\title{
2015 IEEE
}

31st International Conference on Software Maintenance

\section{and Evolution \\ (ICSME)}

Proceedings

Rainer Koschke, Jens Krinke, and Martin Robillard

September 29 - October 1, 2015

Bremen, Germany

Sponsors: IEEE, IEEE Computer Society, TCSE 
2015 IEEE

31st International Conference

on Software Maintenance

and Evolution

(ICSME)

IEEE Catalog Number: CFP15079-ART

ISBN: 978-1-4673-7532-0

Copyright and Reprint Permission:

Abstracting is permitted with credit to the source. Libraries are permitted to photocopy beyond the limit of U.S. copyright law for private use of patrons those articles in this volume that carry a code at the bottom of the first page, provided the per-copy fee indicated in the code is paid through Copyright Clearance Center, 222 Rosewood Drive, Danvers, MA 01923. For other copying, reprint or republication permission, write to IEEE Copyrights Manager, IEEE Operations Center, 445 Hoes Lane, P.O. Box 1331, Piscataway, NJ 08855-1331.

All rights reserved.

Copyright (c) 2015 by the Institute of Electrical and Electronics Engineers

Production: Conference Publishing Consulting, D-94034 Passau, Germany info@conference-publishing.com 


\section{Contents}

\section{Frontmatter}

Messages from the Chairs $\ldots \ldots \ldots \ldots \ldots \ldots \ldots \ldots \ldots \ldots \ldots \ldots \ldots$ iii

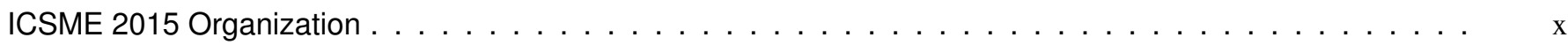

\section{Technical Research Track}

\section{Developers}

Software History under the Lens: A Study on Why and How Developers Examine It

Mihai Codoban, Sruti Srinivasa Ragavan, Danny Dig, and Brian Bailey — Oregon State University, USA; University of

Illinois at Urbana-Champaign, USA . . . . . . . . . . . . . . . . . . . . . . . . . . .

To Fix or to Learn? How Production Bias Affects Developers' Information Foraging during Debugging

David Piorkowski, Scott D. Fleming, Christopher Scaffidi, Margaret Burnett, Irwin Kwan, Austin Z. Henley, Jamie Macbeth, Charles Hill, and Amber Horvath — Oregon State University, USA; University of Memphis, USA; Clemson

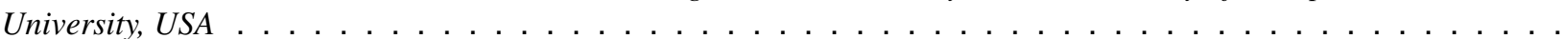

Developers' Perception of Co-change Patterns: An Empirical Study

Luciana L. Silva, Marco Tulio Valente, Marcelo de A. Maia, and Nicolas Anquetil - Federal University of Minas Gerais,

Brazil; Federal University of Uberlândia, Brazil; INRIA, France . . . . . . . . . . . . . . . . . . . . . . . . .

When and Why Developers Adopt and Change Software Licenses

Christopher Vendome, Mario Linares-Vásquez, Gabriele Bavota, Massimiliano Di Penta, Daniel M. German, and Denys Poshyvanyk - College of William and Mary, USA; Free University of Bolzano, Italy; University of Sannio, Italy; University of Victoria, Canada

\section{Program Comprehension}

Investigating Naming Convention Adherence in Java References

Simon Butler, Michel Wermelinger, and Yijun Yu — Open University, UK . . . . . . . . . . . . . . . .

Developing a Model of Loop Actions by Mining Loop Characteristics from a Large Code Corpus

Xiaoran Wang, Lori Pollock, and K. Vijay-Shanker — University of Delaware, USA . . . . . . . . . . . . .

Delta Extraction: An Abstraction Technique to Comprehend Why Two Objects Could Be Related

Naoya Nitta and Tomohiro Matsuoka — Konan University, Japan ． . . . . . . . . . . . . . . . . . . . .

Modeling Changeset Topics for Feature Location

Christopher S. Corley, Kelly L. Kashuda, and Nicholas A. Kraft - University of Alabama, USA; ABB Corporate

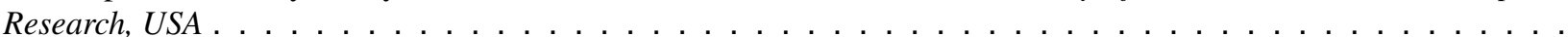

\section{Software Quality}

Four Eyes Are Better Than Two: On the Impact of Code Reviews on Software Quality

Gabriele Bavota and Barbara Russo - Free University of Bolzano, Italy . . . . . . . . . . . . . . .

A Comparative Study on the Bug-Proneness of Different Types of Code Clones

Manishankar Mondal, Chanchal K. Roy, and Kevin A. Schneider - University of Saskatchewan, Canada . . . . . .

An Empirical Study of Bugs in Test Code

Arash Vahabzadeh, Amin Milani Fard, and Ali Mesbah — University of British Columbia, Canada . . . . . . . . .

Investigating Code Review Quality: Do People and Participation Matter?

Oleksii Kononenko, Olga Baysal, Latifa Guerrouj, Yaxin Cao, and Michael W. Godfrey - University of Waterloo,

Canada; Université de Montréal, Canada; École de Technologie Supérieure, Canada

\section{Modularity}

Inter-smell Relations in Industrial and Open Source Systems: A Replication and Comparative Analysis

Aiko Yamashita, Marco Zanoni, Francesca Arcelli Fontana, and Bartosz Walter - Oslo and Akershus University College of Applied Sciences, Norway; University of Milano-Bicocca, Italy; Poznan University of Technology, Poland . . . . . 
Evaluating Clone Detection Tools with BigCloneBench

Jeffrey Svajlenko and Chanchal K. Roy — University of Saskatchewan, Canada . . . . . . . . . . . . . . .

Uncovering Dependence Clusters and Linchpin Functions

David Binkley, Árpád Beszédes, Syed Islam, Judit Jász, and Béla Vancsics — Loyola University Maryland, USA;

University of Szeged, Hungary; University of East London, UK . . . . . . . . . . . . . . . . . . . .

Forked and Integrated Variants in an Open-Source Firmware Project

Ștefan Stănciulescu, Sandro Schulze, and Andrzej Wąsowski - IT University of Copenhagen, Denmark; TU Braun-

schweig, Germany . . . . . . . . . . . . . . . . . . . . . . . . . . .

\section{Program Analysis}

Towards Automating Dynamic Analysis for Behavioral Design Pattern Detection

Andrea De Lucia, Vincenzo Deufemia, Carmine Gravino, and Michele Risi - University of Salerno, Italy . . . . . .

Practical and Accurate Pinpointing of Configuration Errors using Static Analysis

Zhen Dong, Artur Andrzejak, and Kun Shao - University of Heidelberg, Germany; Hefei University of Technology,

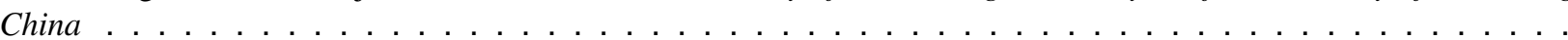

Deterministic Dynamic Race Detection Across Program Versions

Sri Varun Poluri and Murali Krishna Ramanathan — Indian Institute of Science, India . . . . . . . . . . . . .

Program Specialization and Verification using File Format Specifications

Raveendra Kumar Medicherla, Raghavan Komondoor, and S. Narendran - Tata Consultancy Services, India; Indian

Institute of Science, India . . . . . . . . . . . . . . . . . . . . . . . .

\section{Refactoring}

An Empirical Evaluation of Ant Build Maintenance using Formiga

Ryan Hardt and Ethan V. Munson - University of Wisconsin-Eau Claire, USA; University of Wisconsin-Milwaukee, USA

Scripting Parametric Refactorings in Java to Retrofit Design Patterns

Jongwook Kim, Don Batory, and Danny Dig - University of Texas at Austin, USA; Oregon State University, USA . .

System Specific, Source Code Transformations

Gustavo Santos, Nicolas Anquetil, Anne Etien, Stéphane Ducasse, and Marco Tulio Valente — INRIA, France; Federal

University of Minas Gerais, Brazil ． . . . . . . . . . . . . . . . . . . . . .

A Decision Support System to Refactor Class Cycles

Tosin Daniel Oyetoyan, Daniela Soares Cruzes, and Christian Thurmann-Nielsen — NTNU, Norway; SINTEF, Norway;

EVRY, Norway . . . . . . . . . . . . . . . . . . . . . . . . . . . . . . . .

\section{Code Mining and Recommendation}

On the Role of Developer's Scattered Changes in Bug Prediction

Dario Di Nucci, Fabio Palomba, Sandro Siravo, Gabriele Bavota, Rocco Oliveto, and Andrea De Lucia - University of

Salerno, Italy; University of Molise, Italy; Free University of Bolzano, Italy

How Do Developers React to API Evolution? The Pharo Ecosystem Case

André Hora, Romain Robbes, Nicolas Anquetil, Anne Etien, Stéphane Ducasse, and Marco Tulio Valente - Federal University of Minas Gerais, Brazil; University of Chile, Chile; INRIA, France . . . . . . . . . . . . . . .

Who Should Review This Change?: Putting Text and File Location Analyses Together for More Accurate Recommendations

Xin Xia, David Lo, Xinyu Wang, and Xiaohu Yang — Zhejiang University, China; Singapore Management University, Singapore . . . . . . . . . . . . . . . . . . . . . . . .

Exploring API Method Parameter Recommendations

Muhammad Asaduzzaman, Chanchal K. Roy, Samiul Monir, and Kevin A. Schneider - University of Saskatchewan,

Canada . . . . . . . . . . . . . . . . . . . . . . . . . . . . .

\section{Mobile Applications}

How Can I Improve My App? Classifying User Reviews for Software Maintenance and Evolution

Sebastiano Panichella, Andrea Di Sorbo, Emitza Guzman, Corrado A. Visaggio, Gerardo Canfora, and Harald C. Gall University of Zurich, Switzerland; University of Sannio, Italy; TU München, Germany . . . . . . . . . . . . . . . 
User Reviews Matter! Tracking Crowdsourced Reviews to Support Evolution of Successful Apps

Fabio Palomba, Mario Linares-Vásquez, Gabriele Bavota, Rocco Oliveto, Massimiliano Di Penta, Denys Poshyvanyk, and Andrea De Lucia - University of Salerno, Italy; College of William and Mary, USA; Free University of Bolzano,

Italy; University of Molise, Italy; University of Sannio, Italy ～. . . . . . . . . . . . . . . . . .

What Are the Characteristics of High-Rated Apps? A Case Study on Free Android Applications

Yuan Tian, Meiyappan Nagappan, David Lo, and Ahmed E. Hassan — Singapore Management University, Singapore;

Rochester Institute of Technology, USA; Queen's University, Canada . . . . . . . . . . . . . . . . . . . . . . .

GreenAdvisor: A Tool for Analyzing the Impact of Software Evolution on Energy Consumption

Karan Aggarwal, Abram Hindle, and Eleni Stroulia — University of Alberta, Canada . . . . . . . . . . . .

\section{Tool Demo Track}

apiwave: Keeping Track of API Popularity and Migration

André Hora and Marco Tulio Valente — Federal University of Minas Gerais, Brazil . . . . . . . . . . . . . . .

Urbanlt: Visualizing Repositories Everywhere

Andrea Ciani, Roberto Minelli, Andrea Mocci, and Michele Lanza - University of Lugano, Switzerland . . . . .

ePadEvo: A Tool for the Detection of Behavioral Design Patterns

Andrea De Lucia, Vincenzo Deufemia, Carmine Gravino, Michele Risi, and Ciro Pirolli - University of Salerno, Italy

PARC: Recommending API Methods Parameters

Muhammad Asaduzzaman, Chanchal K. Roy, and Kevin A. Schneider - University of Saskatchewan, Canada . . .

ArchFLoc: Locating and Explaining Architectural Features in Running Web Applications

Yan Gao and Daqing Hou — Clarkson University, USA . . . . . . . . . . . . . . . . . .

WSDarwin: A Web Application for the Support of REST Service Evolution

Marios Fokaefs, Mihai Oprescu, and Eleni Stroulia - University of Alberta, Canada . . . . . . . . . . . . 336

DUM-Tool

Simone Romano and Giuseppe Scanniello - University of Basilicata, Italy ～. . . . . . . . . . . . .

\section{Industry Track}

\section{Industry Experience}

An Empirical Study on the Handling of Crash Reports in a Large Software Company: An Experience Report Abdou Maiga, Abdelwahab Hamou-Lhadj, Mathieu Nayrolles, Korosh Koochekian-Sabor, and Alf Larsson — Concordia

University, Canada; Ericsson, Sweden ． . . . . . . . . . . . . . . . . . .

How Developers Detect and Fix Performance Bottlenecks in Android Apps

Mario Linares-Vásquez, Christopher Vendome, Qi Luo, and Denys Poshyvanyk - College of William and Mary, USA

Challenges for Maintenance of PLC-Software and Its Related Hardware for Automated Production Systems: Selected Industrial Case Studies

Birgit Vogel-Heuser, Juliane Fischer, Susanne Rösch, Stefan Feldmann, and Sebastian Ulewicz — TU München, Germany

Code Smells in Spreadsheet Formulas Revisited on an Industrial Dataset

Bas Jansen and Felienne Hermans — Delft University of Technology, Netherlands . . . . . . . . . . . . . . . .

\section{Developer Studies}

Web Usage Patterns of Developers

Christopher S. Corley, Federico Lois, and Sebastián Quezada - ABB Corporate Research, USA; Corvalius, Argentina

Identifying Wasted Effort in the Field via Developer Interaction Data

Gergő Balogh, Gábor Antal, Árpád Beszédes, László Vidács, Tibor Gyimóthy, and Ádám Zoltán Végh — University of

Szeged, Hungary; AENSys Informatics, Hungary . . . . . . . . . . . . . . . . . .

Is This Code Written in English? A Study of the Natural Language of Comments and Identifiers in Practice

Timo Pawelka and Elmar Juergens — TU München, Germany; CQSE, Germany . . . . . . . . . . . . . . . .

Impact Assessment for Vulnerabilities in Open-Source Software Libraries

Henrik Plate, Serena Elisa Ponta, and Antonino Sabetta — SAP Labs, France 


\section{Software Quality}

Experiences from Performing Software Quality Evaluations via Combining Benchmark-Based Metrics Analysis, Software Visualization, and Expert Assessment

Aiko Yamashita - Oslo and Akershus University College of Applied Sciences, Norway . . . . . . . . . . . . .

Do Automatic Refactorings Improve Maintainability? An Industrial Case Study

Gábor Szőke, Csaba Nagy, Péter Hegedús, Rudolf Ferenc, and Tibor Gyimóthy - University of Szeged, Hungary . .

An Empirical Evaluation of the Effectiveness of Inspection Scenarios Developed from a Defect Repository Kiyotaka Kasubuchi, Shuji Morisaki, Akiko Yoshida, and Chikako Ogawa - SCREEN Holdings, Japan; Nagoya University, Japan; Shizuoka University, Japan . . . . . . . . . . . . . . . . . . . . . . . . . . . . . . . . .

Efficient Regression Testing Based on Test History: An Industrial Evaluation Edward Dunn Ekelund and Emelie Engström — Axis Communication, Sweden; Lund University, Sweden . . . . . .

\section{Software Reengineering}

Migrating Legacy Control Software to Multi-core Hardware Michael Wahler, Raphael Eidenbenz, Carsten Franke, and Yvonne-Anne Pignolet - ABB Corporate Research, Switzer-

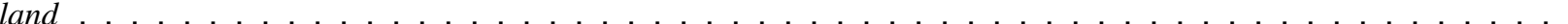

Query by Example in Large-Scale Code Repositories

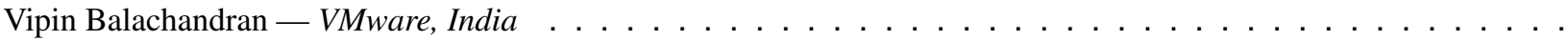

Does Software Modernization Deliver What It Aimed for? A Post Modernization Analysis of Five Software Modernization Case Studies

Ravi Khadka, Prajan Shrestha, Bart Klein, Amir Saeidi, Jurriaan Hage, Slinger Jansen, Edwin van Dis, and Magiel Bruntink — Utrecht University, Netherlands; University of Amsterdam, Netherlands; CGI, Netherlands . . . . . . .

Reverse Engineering a Visual Age Application

Harry M. Sneed and Chris Verhoef — SoRing, Germany; VU University Amsterdam, Netherlands . . . . . . . . . .

Using Static Analysis for Knowledge Extraction from Industrial User Interfaces

Bernhard Dorninger, Josef Pichler, and Albin Kern — Software Competence Center Hagenberg, Austria; Engel Austria,

Austria . . . . . . . . . . . . . . . . . . . . . . . . . . . . . . . .

\section{Early Research Achievements Track}

\section{Defects and Refactoring}

Constrained Feature Selection for Localizing Faults

Tien-Duy B. Le, David Lo, and Ming Li — Singapore Management University, Singapore; Nanjing University, China

Crowdsourced Bug Triaging

Ali Sajedi Badashian, Abram Hindle, and Eleni Stroulia - University of Alberta, Canada . . . . . . . . . . .

Toward Improving Graftability on Automated Program Repair

Soichi Sumi, Yoshiki Higo, Keisuke Hotta, and Shinji Kusumoto - Osaka University, Japan . . . . . . . . . .

Mining Stack Overflow for Discovering Error Patterns in SQL Queries

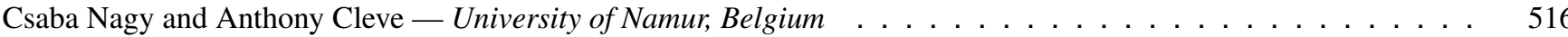

Towards Purity-Guided Refactoring in Java

Jiachen Yang, Keisuke Hotta, Yoshiki Higo, and Shinji Kusumoto - Osaka University, Japan . . . . . . . . . .

Fitness Workout for Fat Interfaces: Be Slim, Clean, and Flexible

Spyros Kranas, Apostolos V. Zarras, and Panos Vassiliadis - University of Ioannina, Greece ～. . . . . . . . . .

\section{Social and Developers}

Choosing Your Weapons: On Sentiment Analysis Tools for Software Engineering Research

Robbert Jongeling, Subhajit Datta, and Alexander Serebrenik - Eindhoven University of Technology, Netherlands;

Singapore University of Technology and Design, Singapore . . . . . . . . . . . . . . . . . . . . .

Assessing Developer Contribution with Repository Mining-Based Metrics

Jalerson Lima, Christoph Treude, Fernando Figueira Filho, and Uirá Kulesza - Federal University of Rio Grande do Norte, Brazil . . . . . . . . . . . . . . . . . . . . . . . . . . . . . . . . . . 
What's Hot in Software Engineering Twitter Space?

Abhishek Sharma, Yuan Tian, and David Lo - Singapore Management University, Singapore . . . . . . . . . . .

Validating Metric Thresholds with Developers: An Early Result

Paloma Oliveira, Marco Tulio Valente, Alexandre Bergel, and Alexander Serebrenik - Federal University of Minas

Gerais, Brazil; University of Chile, Chile; Eindhoven University of Technology, Netherlands . . . . . . . . . . . .

Towards a Survival Analysis of Database Framework Usage in Java Projects

Mathieu Goeminne and Tom Mens — University of Mons, Belgium . . . . . . . . . . . . . . . . . . .

\section{Maintenance and Analysis}

Exploring the Use of Deep Learning for Feature Location Christopher S. Corley, Kostadin Damevski, and Nicholas A. Kraft - University of Alabama, USA; Virginia Commonwealth University, USA; ABB Corporate Research, USA

Using Stereotypes in the Automatic Generation of Natural Language Summaries for $\mathrm{C}_{++}$Methods

Nahla J. Abid, Natalia Dragan, Michael L. Collard, and Jonathan I. Maletic - Kent State University, USA; University of

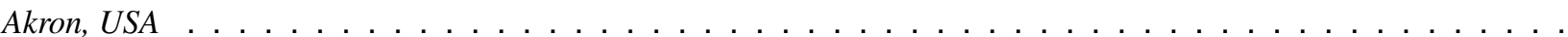

Keecle: Mining Key Architecturally Relevant Classes using Dynamic Analysis

Liliane do Nascimento Vale and Marcelo de A. Maia — Federal University of Uberlândia, Brazil . . . . . . . . .

Combining Software Interrelationship Data across Heterogeneous Software Repositories

Nikola Ilo, Johann Grabner, Thomas Artner, Mario Bernhart, and Thomas Grechenig - Vienna University of Technology,

Recovering Transitive Traceability Links among Software Artifacts

Kazuki Nishikawa, Hironori Washizaki, Yoshiaki Fukazawa, Keishi Oshima, and Ryota Mibe - Waseda University,

Japan; Hitachi, Japan . . . . . . . . . . . . . . . . . . . . . . . . . . . . . . . . . .

Live Object Exploration: Observing and Manipulating Behavior and State of Java Objects

Benjamin Biegel, Benedikt Lesch, and Stephan Diehl — University of Trier, Germany . . . . . . . . . . . .

\section{Doctoral Symposium}

\section{Post-Doctoral}

Supporting Newcomers in Software Development Projects

Sebastiano Panichella - University of Zurich, Switzerland . . . . . . . . . . . . . . . . .

Advances in Software Product Quality Measurement and Its Applications in Software Evolution

Péter Hegedús — University of Szeged, Hungary ～. . . . . . . . . . . . . . . . . . . . .

\section{Pre-Doctoral}

Treating Software Quality as a First-Class Entity

Yuriy Tymchuk - University of Lugano, Switzerland .

Detection Strategies of Smells in Web Software Development

Maurício F. Aniche - University of São Paulo, Brazil ． . . . . . . . . . . . . . . . . . . . . . .

Code Smells in Highly Configurable Software

Wolfram Fenske - University of Magdeburg, Germany . . . . . . . . . . . . . . . . . . .

A Model-Based Approach to Software Refactoring

Ioana Verebi - Politehnica University of Timisoara, Romania 